\title{
Polygenic risk for schizophrenia and measured domains of cognition in individuals with psychosis and controls
}

Rebecca Shafee ${ }^{1,2}$, Pranav Nanda ${ }^{3}$, Jaya L. Padmanabhan ${ }^{4}$, Neeraj Tandon, Ney Alliey-Rodriguez ${ }^{6}$, Sreeja Kalapurakkel ${ }^{2,7,8}$, Daniel J. Weiner ${ }^{2,7,8,9}$, Raquel E. Gur ${ }^{10}$, Richard S. E. Keefe ${ }^{11}$, Scot K. Hill12, Jeffrey R. Bishop ${ }^{13,14}$, Brett A. Clementz ${ }^{15}$, Carol A. Tamminga ${ }^{16}$, Elliot S. Gershon $\mathbb{1 0}^{6,17}$, Godfrey D. Pearlson ${ }^{18}$, Matcheri S. Keshavan ${ }^{19}$, John A. Sweeney ${ }^{20}$, Steven A. McCarroll ${ }^{1,2,7}$ and Elise B. Robinson $2,7,8,21$

\begin{abstract}
Psychotic disorders including schizophrenia are commonly accompanied by cognitive deficits. Recent studies have reported negative genetic correlations between schizophrenia and indicators of cognitive ability such as general intelligence and processing speed. Here we compare the effect of polygenetic risk for schizophrenia (PRS $S_{s c z}$ on measures that differ in their relationships with psychosis onset: a measure of current cognitive abilities (the Brief Assessment of Cognition in Schizophrenia, BACS) that is greatly reduced in psychotic disorder patients, a measure of premorbid intelligence that is minimally affected by psychosis onset (the Wide-Range Achievement Test, WRAT); and educational attainment (EY), which covaries with both BACS and WRAT. Using genome-wide single nucleotide polymorphism (SNP) data from 314 psychotic and 423 healthy research participants in the Bipolar-Schizophrenia Network for Intermediate Phenotypes (B-SNIP) Consortium, we investigated the association of PRS SCZ with BACS, WRAT, and EY. Among apparently healthy individuals, greater genetic risk for schizophrenia ( $\mathrm{PRS}_{\mathrm{SCZ}}$ ) was significantly associated with lower BACS scores $\left(r=-0.17, p=6.6 \times 10^{-4}\right.$ at $\left.\mathrm{P}_{\mathrm{T}}=1 \times 10^{-4}\right)$, but not with WRAT or EY. Among individuals with psychosis, $\mathrm{PRS}_{\mathrm{SCZ}}$ did not associate with variations in any of these three phenotypes. We further investigated the association between PRS $_{s c z}$ and WRAT in more than 4500 healthy subjects from the Philadelphia Neurodevelopmental Cohort. The association was again null $(p>0.3, N=4511)$, suggesting that different cognitive phenotypes vary in their etiologic relationship with schizophrenia.
\end{abstract}

\section{Introduction}

Schizophrenia is a debilitating psychiatric disorder that commonly involves severe cognitive deficits that compromise functional ability ${ }^{1,2}$. Underperformance in general intelligence tasks as well as tasks designed to be specific to cognitive domains such as memory, executive function, and motor function have been noted in psychosis patients ${ }^{3}$.

\footnotetext{
Correspondence: Rebecca Shafee (rebecca_shafee@hms.harvard.edu) 'Department of Genetics, Harvard Medical School, Boston, MA, USA

${ }^{2}$ Stanley Center for Psychiatric Research, Broad Institute of MIT and Harvard, Cambridge, MA, USA

Full list of author information is available at the end of the article
}

Many schizophrenia-associated cognitive deficits are present many years prior to the onset of the illness ${ }^{4,5}$. A meta-analysis of 4396 schizophrenia cases and 745,000 controls showed that every point decrease in premorbid IQ associated with a $3.7 \%$ increase in schizophrenia risk ${ }^{6}$. In a nationwide cohort of over 900,000 Swedish individuals, children with the lowest grades showed a 4-fold increased risk of developing schizophrenia and schizoaffective disorder and a 3-fold increased risk of developing other psychotic illnesses ${ }^{7}$. Additionally, studies of clinically high-risk (CHR) groups have shown that people with attenuated psychotic symptoms were cognitively impaired compared to healthy controls $(\mathrm{HC})$ and that, 
within the CHR group, those that converted to a chronic psychotic disorder within one or 2 years of ascertainment displayed lower cognitive performance compared to those that did not convert ${ }^{8-11}$. Together these results indicate that cognitive deficits are significantly associated with risk of developing a psychotic illness.

Both cognitive performance and psychotic disorders such as schizophrenia are heritable ${ }^{12-19}$, and significant genetic overlap has been consistently reported between schizophrenia and some indicators of cognitive ability, such as general intelligence or processing speed ${ }^{20-26}$. However, it is still unclear how the genetic differences associated with schizophrenia influence cognitive function, and which domains of cognitive function are most associated with schizophrenia risk.

Motivated by these earlier findings, we investigated the relationship between polygenic risk for schizophrenia-as defined by large constellations of common variants that associate with schizophrenia risk $\left(\mathrm{PRS}_{\mathrm{SCZ}}\right)$-and three cognitive phenotypes in the Bipolar-Schizophrenia Network for Intermediate Phenotypes ${ }^{27,28}$ (B-SNIP) cohort: (1) the Brief Assessment of Cognition in Schizophrenia $(\mathrm{BACS})^{29}$, which provides a composite score of current general cognitive function; (2) the Wide-Range Achievement Test (WRAT) ${ }^{30-32}$ reading score, a measure of premorbid intellectual potential; and (3) educational attainment (as measured by years of education, EY). These phenotypes are correlated but differentially associated with psychosis-spectrum case status. Compared to BACS or general cognition, WRAT scores are minimally affected by psychosis onset ${ }^{8}$, and are commonly used as a measure for premorbid intelligence in people with psychotic disorders $^{30-32}$; educational attainment is phenotypically associated with WRAT and BACS and also strongly genetically overlaps with $\operatorname{cognition}^{33,34}$. A companion analysis was conducted in the large Philadelphia Neurodevelopmental Cohort (PNC, $N=4511)^{35-37}$ investigating the relationship between WRAT and $\mathrm{PRS}_{\mathrm{SCZ}}$ since WRAT measures were also available in the PNC.

As an additional validation analysis we investigated the relationship between the polygenic score of educational attainment (PRS EDUC $_{\text {) }}$ and these three cognitive phenotypes because of the significant genetic overlap between educational attainment and cognition ${ }^{25,34}$.

\section{Methods}

\section{Study design and participants}

Demographic information about the B-SNIP and the PNC cohorts can be found in Table 1. The B-SNIP analysis included 737 Caucasians from the BipolarSchizophrenia Network for Intermediate Phenotypes (B$\mathrm{SNIP})^{27,28}$, which is a five-site consortium (Maryland Psychiatric Research Center, University of Chicago/University of Illinois at Chicago, University of Texas-
Table 1 Demographic information for the B-SNIP and PNC cohorts

\begin{tabular}{|c|c|c|c|c|}
\hline & \multicolumn{3}{|l|}{ B-SNIP } & \multirow{3}{*}{$\begin{array}{l}\text { PNC } \\
\text { Controls }\end{array}$} \\
\hline & \multicolumn{2}{|l|}{ NPSYCH } & \multirow[t]{2}{*}{ PSYCH } & \\
\hline & $\mathrm{HC}$ & NPFAM & & \\
\hline N & 180 & 243 & 314 & 4511 \\
\hline Age (years) & $38.7(12.8)$ & $46.5(14.5)$ & $34.9(1.3)$ & $13.8(3.7)$ \\
\hline Sex $(\% F)$ & 51.6 & 73.7 & 45.9 & 50.0 \\
\hline Years of education & $15.2(2.5)$ & $14.9(2.5)$ & $13.9(2.3)$ & N/A \\
\hline
\end{tabular}

B-SNIP Bipolar-Schizophrenia Network for Intermediate Phenotypes, PNC Philadelphia Neurodevelopmental Cohort, NPSYCH B-SNIP nonpsychotic group consisting of healthy controls (HC) and nonpsychotic relatives (NPFAM), PSYCH B-SNIP psychotic proband group consisting of schizophrenia $(N=100)$, psychotic bipolar $(N=143)$, and schizoaffective disorder $(N=71)$ patients. Mean values are shown with standard deviations in parentheses. Years of Education was not an applicable measure for the young PNC cohort. Only samples with European ancestry were used in this study.

Southwestern, Wayne State University/Harvard University, and the Institute of Living/Yale University) organized to address questions about diagnostic boundaries and familiality of intermediate phenotypes. Previous work using this cohort reported BACS performance to be consistent with a dimensional model of psychosis ${ }^{27,38}$; Hill et al. (2013) showed that cognitive performance declined progressively as affective symptoms became less prominent and psychotic features became more pronounced and pervasive. Due to these findings, we combined all psychotic probands to form the PSYCH group $(N=314)$ consisting of schizophrenia $(N=100)$, psychotic bipolar disorder $(N=143)$, and schizoaffective disorder patients $(N=71)$. The NPSYCH group consisted of unrelated nonpsychotic individuals combining samples collected as controls $(\mathrm{HC}, N=180)$ and first-degree relatives of probands with no history of psychosis (NPFAM, $N=243$ ) and without elevated axis II traits ${ }^{27}$ (cluster A or cluster B). While the NPFAM members of the NPSYCH group were related to probands in the PSYCH group, none of the analyses included related individuals (e.g., group differences were calculated between HC and PSYCH or between NPFAM and HC; correlation analyses with $\mathrm{PRS}_{\mathrm{SCZ}}, \mathrm{PRS}_{\mathrm{EDUC}}$ or between the three cognitive phenotypes were conducted within the PSYCH and the NPSYCH groups separately). All participants provided written informed consent. Institutional review boards at each site approved the study and all sites used identical diagnostic, clinical, and recruitment techniques $^{28}$.

The Philadelphia Neurodevelopmental Cohort (PNC) is a sample from the greater Philadelphia area, including over 9000 individuals aged 8-21 years who received medical care at the Children's Hospital at Philadelphia 
network $^{35-37}$. The overall inclusion criteria for the cohort included: (1) Ability to provide signed informed consent (parental consent was required for participants under age 18), (2) English language proficiency, and (3) Physical and cognitive ability to participate in computerized cognitive testing. Only unrelated participants (pi-hat $<0.2$ ) of European ancestry were used in this work. Individuals with significant medical conditions that can impact brain function, as well as those with either an invalid or incomplete neurocognitive battery were excluded. After genetic quality control (described below and in Supplementary Material) the final sample for this study consisted of 4511 unrelated individuals (mean age 13.76 years, S.D. 3.66 years). All analyses in the PNC cohort were done in this entire sample.

\section{Cognitive measures}

Three cognitive measures were available in the B-SNIP cohort: BACS, WRAT, and educational attainment. General cognitive function in the B-SNIP was measured by the BACS, which is a $30 \mathrm{~min}$ test of global neuropsychological function ${ }^{29}$. Premorbid intellectual potential was measured using the reading score of the Wide-Range Achievement Test (WRAT IV), which has a phenotypic correlation of $\sim 0.4$ with full-scale intelligent quotient ${ }^{30,39}$. Self-reported years of education completed at the time of recruitment was used as a measure of EY. WRAT was similarly assessed in the PNC sample. A BACS equivalent was not available in the $\mathrm{PNC}$ and due to the young age of the subjects (8-21 years) EY would be largely redundant to age itself.

\section{Genetic analyses}

Genetic data for the B-SNIP project were collected for 2053 subjects (multi-ethnic sample) using the Illumina Infinium PsychArray BeadChip ${ }^{\text {tw }}$ platform. Genotypes underwent quality control using PLINK $1.9^{40,41}$ based on a standardized protocol ${ }^{42}$ (Supplementary Material). After initial quality control, and removal of individuals with missing cognitive phenotypes, 1528 samples remained of whom 927 were self-reported Caucasians (SRC). To avoid population stratification, only SRC samples were used in all analyses. The ancestries of these SRC samples were verified by principal component analysis combining the BSNIP genotype data with the 1000 Genomes phase 1 data $^{43}$. Samples that were more than four standard deviations away from the SRC group mean along the first ten principal components were excluded resulting in a final sample size of 737 (Figure S1). Imputation of the BSNIP genetic data was performed using HAPI-UR for prephasing $^{44}$ and IMPUTE2 for imputation ${ }^{45,46}$ using a multi-ethnic (the 1000 Genomes phase 1 reference panel $^{43}$ ) reference panel ${ }^{47}$. Poorly imputed single nucleotide polymorphisms (SNPs) were filtered post- imputation (SNPs with information score $<0.5^{48}$ were removed) resulting in 22.5 million imputed SNPs.

Genotype data for 8211 multi-ethnic PNC samples were downloaded from dbGAP. These data were distributed across five different Illumina genotyping chips (as described in the Supplementary Material). Quality control was performed with the programs PLINK ${ }^{41}$ and GCTA ${ }^{49}$. After principal component analysis of the PNC data combined with the HapMap reference panel ${ }^{50}$, only samples with European ancestry were retained by visual inspection (overlapping with CEU and TSI, Figure S2). Following these steps 4733 samples and 204,597 markers were retained for imputation. The Michigan Imputation Server ${ }^{51}$ was used for genetic imputation of the PNC data (Minimac $3^{51}$ for imputation and HAPI-UR ${ }^{44}$ for phasing) with the 1000 genome phase 3 data $^{52}$ as reference panel resulting in a total of 18 million imputed markers. The imputed variants were filtered for info score $\geq 0.6$ (7.9 million markers) for polygenic score calculation with PLINK. Filtering samples for medical criteria and missing cognitive phenotypes (see Study Design and Participants) resulted in a final PNC sample of 4511 unrelated healthy individuals.

Schizophrenia polygenic profile scores $\left(\mathrm{PRS}_{\mathrm{SCZ}}\right)$ and educational attainment polygenic scores ( $\mathrm{PRS}_{\mathrm{EDUC}}$ ) were calculated using the schizophrenia GWAS summary statistics of the Psychiatric Genome Consortium (PGC) ${ }^{19}$ (https://www.med.unc.edu/pgc/results-and-downloads) and the summary statistics from Okbay et $\mathrm{al}^{34}$, respectively. Score calculation was done using custom scripts in the B-SNIP and using PLINK in the PNC. Of the 120,636 PGC schizophrenia polygenic score training SNPs, 101,927 overlapped with the imputed B-SNIP data and 85,598 overlapped with the imputed PNC data. Of the 626,000 educational attainment GWAS markers (clumped using the 1000 Genome $^{43}$ European Ancestry group; $r^{2}<$ 0.1 within a $500 \mathrm{~kb}$ window of a more significantly associated SNP), 530,894 and 210,501 SNPs were in common with the imputed data in B-SNIP and the PNC, respectively. Polygenic scores were calculated for seven $p$-value thresholds of significance of association: $P \leq 10^{-4}, 0.001$, $0.01,0.05,0.1,0.5$, and 1.0 . The first 10 principal components from ancestry analyses of B-SNIP and PNC were used as covariates for correlation analyses in the respective cohorts.

\section{Statistical analyses}

All statistical analyses in B-SNIP were performed using Matlab (version 2012b). Correlations between BACS, WRAT, and EY and the polygenic scores were calculated within the PSYCH group and the NPSYCH group ( $\mathrm{HC}+$ NPFAM) separately using the Spearman Rank method, which is a nonparametric measure of correlation (deviation from normal distribution was noted in WRAT, EY, 
and $\mathrm{PRS}_{\mathrm{SCZ}}$ in specific groups). Age, sex, data collection site, the first 10 principal components from the genetic ancestry analysis, and DSM diagnosis (schizophrenia/ bipolar disorder/schizoaffective disorder status for members of the PSYCH group and respective relative's diagnosis for member's of the NPFAM group) were regressed out for correlation analyses within each group. As an additional precaution, the samples' HC/NPFAM status was used as a covariate for all analyses within the NPSYCH group. Differences in BACS, WRAT, and EY (Figure S3) between the HC, PSYCH, and NPFAM groups were calculated using the Kruskal-Wallis test (a nonparametric method for testing whether samples originate from the same distribution, which was used due to unequal variances in BACS between groups) after regressing out the effects of age, sex, data collection site, and the first ten principal components from the genetic ancestry analysis. These group differences were calculated between $\mathrm{HC} / \mathrm{PSYCH}$ and HC/NPFAM instead of $\mathrm{NPSYCH} / \mathrm{PSYCH}$ so that only unrelated individuals were compared. This was not a concern for correlation analyses within the NPSYCH group since the HC and the NPFAM subgroups were unrelated. Group differences in $\mathrm{PRS}_{\mathrm{SCZ}}$ and $\mathrm{PRS}_{\text {EDUC }}$ were calculated between the $\mathrm{HC}$ and PSYCH groups (Fig. 1, Table S1, Kruskal-Wallis test was used due to unequal variance between groups for PRS $_{\text {EDUC }}$ ) after regressing out the effects of data collection site and the first ten ancestry principal components. To

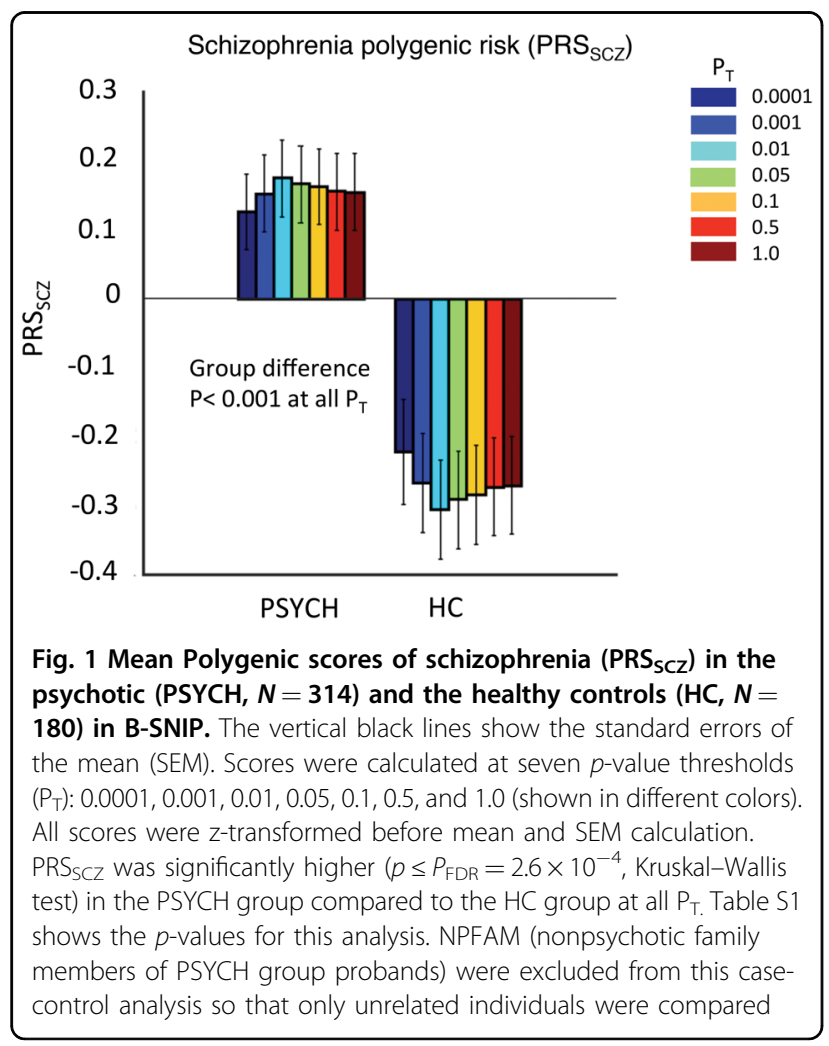

correct for multiple hypotheses testing in analyses of the B-SNIP cohort a false discovery rate (FDR) approach ${ }^{53}$ was used following the example of recent studies that used polygenic risk scores ${ }^{25,54}$. For analyses with polygenic scores in B-SNIP the combined $\mathrm{P}_{\mathrm{FDR}-\mathrm{PRS}}$ was 0.0064 at $\alpha=0.05$. Analysis specific FDR $p$-values are reported with each result.

All analyses in the PNC were done using RStudio ${ }^{55}$ (Version 1.0.44). Since individuals in the PNC sample were controls and unrelated, correlations between polygenic scores and WRAT were calculated within the entire sample controlling for effects of age, sex, and the first 10 ancestry principal components using the Spearman Rank method. The FDR-corrected ${ }^{53} p$-value threshold for PNC was $P_{\mathrm{FDR}-\mathrm{PNC}}=3.1 \times 10^{-12}$ at $\alpha=0.05$ for all analyses using $\mathrm{PRS}_{\mathrm{SCZ}}$ and $\mathrm{PRS}_{\mathrm{EDUC}}$.

\section{Results}

Genetic risk for schizophrenia was higher among individuals with psychosis in the mixed diagnostic group in B-SNIP

An individual's polygenic risk of schizophrenia, $\mathrm{PRS}_{\mathrm{SCZ}}$, estimates genome-wide common genetic influences on the risk of developing schizophrenia. Compared to the $\mathrm{HC}$ (Fig. 1), individuals with psychosis from 3 diagnosis groups in the B-SNIP sample (schizophrenia, psychotic bipolar, schizoaffective disorder) showed significantly higher $\mathrm{PRS}_{\mathrm{SCZ}}\left(p \leq \mathrm{P}_{\mathrm{FDR}}=2.6 \times 10^{-4}\right.$, Table S1 $)$ at all $\mathrm{P}_{\mathrm{T}}$. Among the psychosis probands schizophrenia patients had highest $\mathrm{PRS}_{\mathrm{SCZ}}$ (Figure S4). In our sample PRS $\mathrm{EDUC}_{\mathrm{E}}$ did not differ significantly between the PSYCH and the HC groups (Table S1). Figure S4 shows the distributions of $\mathrm{PRS}_{\mathrm{SCZ}}$ and $\mathrm{PRS} \mathrm{EDUC}_{\mathrm{E}}$ for the different DSM diagnosis groups.

Psychosis did not alter the correlations between EY, BACS, and WRAT in B-SNIP

An individual's educational attainment, cognitive functioning and intellectual potential are interdependent traits $^{56}$. We examined these relationships within the PSYCH and the NPSYCH groups separately in the BSNIP sample and found that the presence of psychosis did not alter the extent to which the phenotypes are independent (Fig. 2). Although BACS, WRAT, and EY were significantly lower in the PSYCH group compared to the HC group (Figure S3), the effect size of deficit in BACS (Cohen's $d=1.24, p=8.1 \times 10^{-32}$ ) was more than three times greater than that of EY or WRAT. Additionally, partial correlation analyses between pairs of these three phenotypes controlling for the third phenotype revealed that, (1) EY and WRAT shared a positive correlation that could not be accounted for by BACS; (2) WRAT and BACS shared a positive correlation that could not be accounted for by EY; and (3) although EY and BACS were 
weakly positively correlated, this correlation was mediated via factors that could be captured by WRAT (Table S2).

Higher polygenetic risk for schizophrenia was significantly associated with lower BACS scores, but not WRAT or EY in nonpsychotic individuals in B-SNIP

To evaluate whether the genetic risk for schizophrenia associates with variations in BACS, EY, and WRAT, correlations of $\mathrm{PRS}_{\mathrm{SCZ}}$ with these measures were calculated within the PSYCH and the NPSYCH groups in the B-SNIP sample separately. Figure 3 shows the strongest correlations for each phenotype. The numerical values for the correlation coefficients and the $p$-values for both groups at all $\mathrm{P}_{\mathrm{T}}$ can be found in Table S3. BACS showed

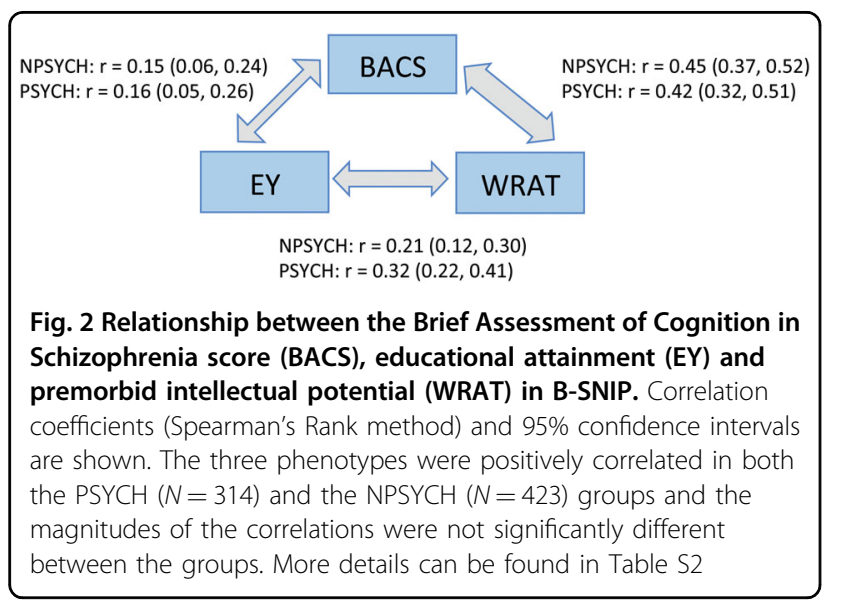

significant negative association with $\mathrm{PRS}_{\mathrm{SCZ}}$ in the NPSYCH group (Fig. 3a, $r=-0.17$ and $p=6.6 \times 10^{-4}$ at $P_{\mathrm{T}}=1 \times 10^{-4}$ ), but not in the PSYCH group. This association remained significant when variability due to EY and WRAT were accounted for by additionally controlling for those two phenotypes (Table S3). Nominally significant $(p<0.05)$ negative association was seen between $\mathrm{PRS}_{\mathrm{SCZ}}$ and EY in the NPSYCH group, but not in the PSYCH group (Fig. 3b). WRAT was not significantly or nominally associated with $\mathrm{PRS}_{\mathrm{SCZ}}$ in either group.

The correlation between BACS and PRS $\mathrm{SCZ}_{\mathrm{S}}$ within the schizophrenia proband group only (SZP, $N=100$ ) was also not significant, similar to the results of the entire PSYCH group. Adding illness duration, number of hospitalization, chlorpromazine dose equivalent, number of psychotropic drugs, and social-functional scale score as covariates in the correlation analysis between BACS and $\mathrm{PRS}_{\mathrm{SCZ}}$ did not alter the lack of significant results in the PSYCH group.

Since the NPSYCH group consisted of nonpsychotic individuals recruited as $\mathrm{HC}$ as well as the nonpsychotic family members of the psychosis probands (NPFAM, all subjects within this group were unrelated), the significance of the association of $\mathrm{PRS}_{\mathrm{SCZ}}$ with BACS was additionally investigated within the $\mathrm{HC}$ and the NPFAM groups individually (Supplementary Material). At the subgroup level, statistically significant correlation (Table S4) between BACS and $\mathrm{PRS}_{\mathrm{SCZ}}$ was seen at $P_{\mathrm{T}}=10^{-4}$ in the HC group $\left(r=-0.25, p=1.9 \times 10^{-3}\right)$, which remained significant when EY and WRAT were regressed out. In the NPFAM subgroup, significant

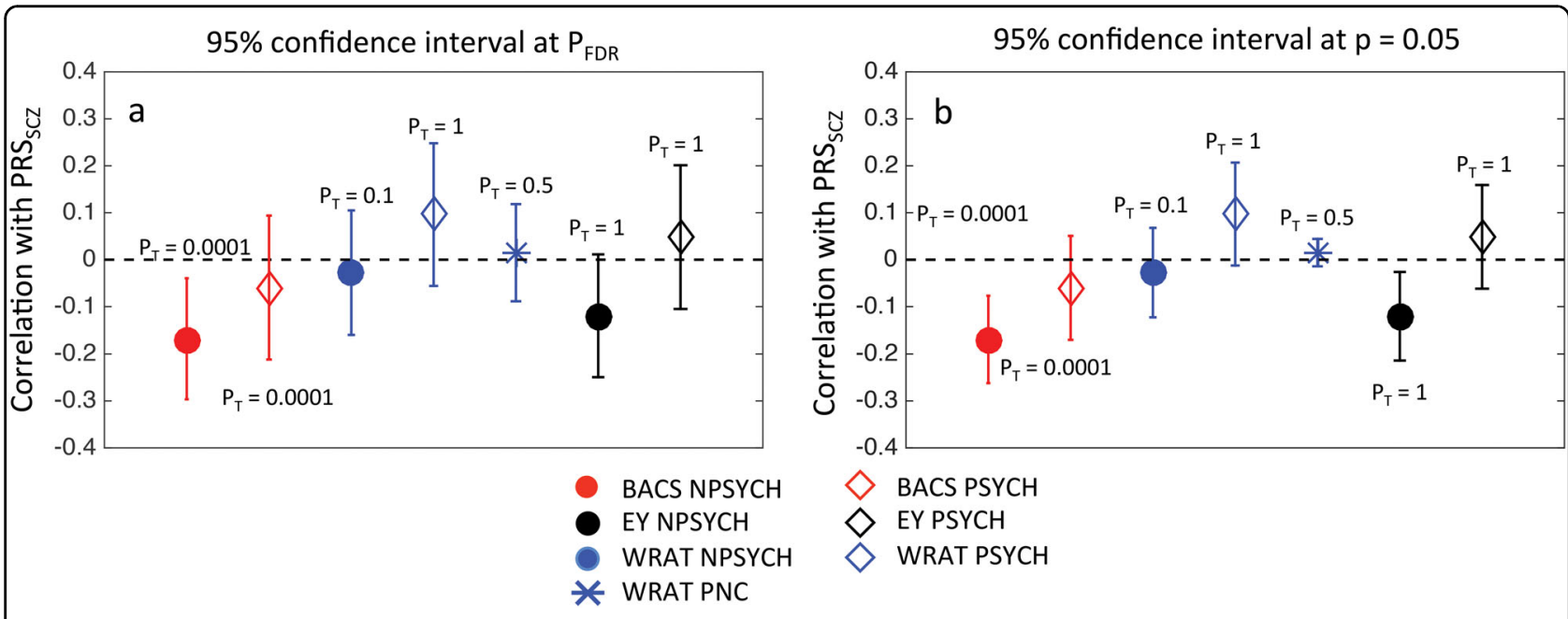

Fig. 3 Correlations of the polygenic risk of schizophrenia ( $\mathrm{PRS}_{\mathrm{SCZ}}$ ) with the Brief Assessment of Cognition in Schizophrenia score (BACS), premorbid intellectual potential (WRAT) and educational attainment (EY). For B-SNIP: $N=314$ (PSYCH), $N=423$ (NPSYCH) and for the PNC: $N$ $=4511$. All markers other than the blue star, which represents PNC, show results for B-SNIP. Correlation coefficients are shown with $95 \%$ confidence intervals for FDR-corrected $p$-value threshold $\left(P_{\mathrm{FDR}-\mathrm{B}-\mathrm{SNIP}}=0.0064, P_{\mathrm{FDR}-\mathrm{PNC}}=3.1 \times 10^{-12}\right)(\mathbf{a})$ and $p=0.05(\mathbf{b})$. Only the strongest correlation (Spearman's Rank method) for each phenotype is shown with the corresponding $\mathrm{P}_{\mathrm{T}}$ labeled. Correlation coefficients and corresponding $p$-values for all $\mathrm{P}_{\mathrm{T}}$ can be found in Tables S3 and S6 


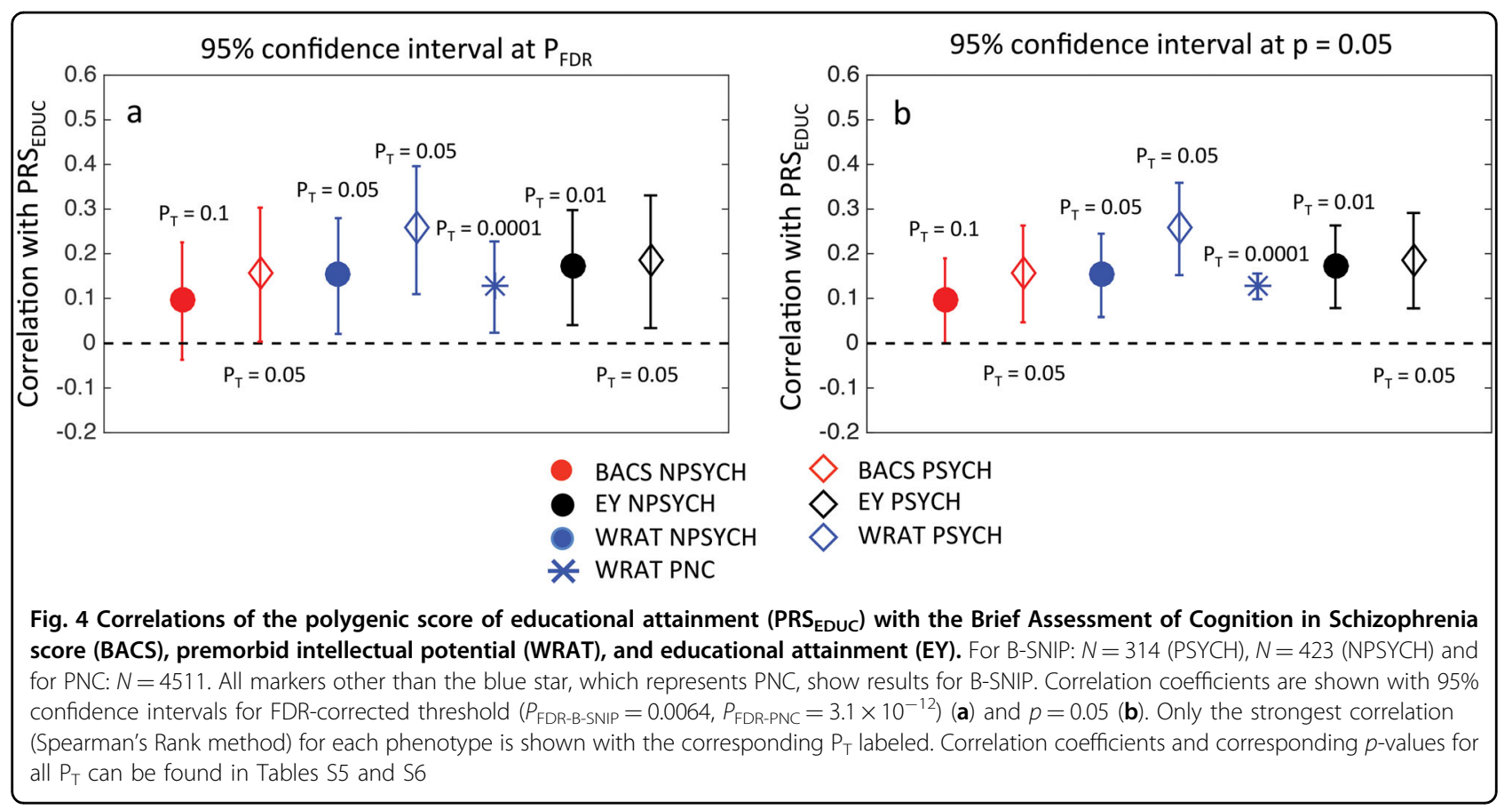

negative correlation was detected at $\mathrm{P}_{\mathrm{T}}=0.01(r=-0.19$, $p=6.4 \times 10^{-3}$ ) when EY and WRAT were regressed out (Table S4).

The polygenic score of educational attainment, PRS $_{\text {EDUC }}$, showed significant positive correlations with EY in both the PSYCH group (Fig. 4, strongest correlation of $r=0.19, p=0.0016$ at $\left.\mathrm{P}_{\mathrm{T}}=0.05\right)$ and the NPSYCH group (Fig. 4, strongest correlation of $r=0.17, p=7 \times 10^{-4}$ at $\left.P_{\mathrm{T}}=0.01\right)$. Significant positive correlations were observed

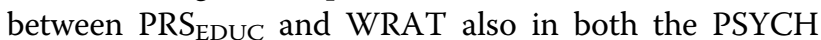
group and the NPSYCH group (Fig. 4) at several $\mathrm{P}_{\mathrm{T}}$ (strongest correlation of $r=0.26, p=1.1 \times 10^{-5}$ at $P_{\mathrm{T}}=0.05$ in PSYCH and strongest correlation of $r=0.15$, $p=2.4 \times 10^{-3}$ at $P_{\mathrm{T}}=0.05$ in NPSYCH). No significant correlation was found between $\mathrm{PRS}_{\mathrm{EDUC}}$ and BACS in either group. The numerical values for all the correlation coefficients and $p$-values can be found in Table S5.

\section{Polygenic risk for schizophrenia and WRAT were unrelated in the PNC}

We investigated the relationship between $\mathrm{PRS}_{\mathrm{SCZ}}$, PRS $_{\text {EDUC, and WRAT in unrelated healthy individuals }}$ from the Philadelphia Neurocognitive Cohort $(N=4511)$. Our analyses showed lack of significant association between $\mathrm{PRS}_{\mathrm{SCZ}}$ and WRAT at all $\mathrm{P}_{\mathrm{T}}$ (Table S6, Fig. 3, $p>0.3$ at all $\mathrm{P}_{\mathrm{T}}$ ) and significant positive association between PRS $_{\text {EDUC }}$ and WRAT at all $\mathrm{P}_{\mathrm{T}}$ (Fig. 4, Table S6, $p \leq P_{\text {FDR-PNC }}=3.1 \times 10^{-12}$ at all $\mathrm{P}_{\mathrm{T}}$; maximum correlation of $r=0.13$ and $p<2.2 \times 10^{-16}$ at $\left.P_{\mathrm{T}}=1 \times 10^{-4}\right)$. These results were consistent with the findings in the B-SNIP cohort.

\section{Discussion}

Cognitive deficits are widespread in psychotic disorder patients, especially in schizophrenia. Recent molecular genetics studies have shown that schizophrenia is genetically negatively correlated with multiple measures of cognition ${ }^{25,57}$. Our work focused on the relationship between the common polygenic risk of schizophrenia and three cognitive measures that are phenotypically correlated (Fig. 2), but differentially associated (Fig S3) with psychosis-spectrum case status. Our main findings were: (1) BACS, a measure of general cognitive function (most strongly affected in the patient group) was negatively associated with the polygenic risk of schizophrenia in apparently healthy individuals, (2) WRAT, often used as a measure of premorbid intelligence in psychosis-spectrum patients, was not associated with the common genetic risk of schizophrenia in healthy or psychotic individuals, and (3) the negative association between BACS and the polygenic risk of schizophrenia did not appear to hold in the psychotic patient group.

The first finding is consistent with other recent reports of genetic overlap between general cognitive function and schizophrenia ${ }^{24,25,57}$. For example, Trampush et al. ${ }^{57}$, reported a genetic correlation of -0.17 between schizophrenia and general intelligence. Our B-SNIP sample was not large enough for applying the recently developed methods of LD Score Regression ${ }^{58}$ or GCTA $^{49}$ to calculate genetic correlation between traits. However, PRS correlations to phenotype are mathematically translatable to genetic correlations between two traits ${ }^{59}$. In other words, a positive PRS association would translate to a 
positive genetic correlation of estimable magnitude. Hence, the negative association between $\mathrm{PRS}_{\mathrm{SCZ}}$ and BACS is consistent with the above-mentioned negative genetic correlations between cognitive measures and schizophrenia $^{25,37}$. Such negative associations have been shown in young cohorts also-Riglin et al. ${ }^{60}$ recently showed that lower performance intelligence quotient is associated with higher common genetic risk of schizophrenia in 14,701 samples of the ALSPAC cohort (age 7-9 years). Also, within the PNC cohort, it has been shown previously that the common genetic risk of schizophrenia negatively influences speed of verbal reasoning and emotion identification ${ }^{39}$.

The second and third findings were intriguing and warrant further investigation. In spite of sharing a phenotypic correlation of 0.4 with BACS, WRAT did not associate even nominally with the genetic risk of schizophrenia in either of our cohorts, including the large PNC. Although both BACS and WRAT measure cognitive function, BACS measures an individual's ability to use cognitive resources to solve problems and WRAT is more of a measure of crystallized verbal knowledge. These results indicate the possibility that cognitive domains measured by BACS-rather than other brain phenotypes that shape premorbid intelligence-may be more direct targets of the genetic risk factors of schizophrenia.

Though we observed a significant negative correlation between $\mathrm{PRS}_{\mathrm{SCZ}}$ and BACS at multiple $\mathrm{P}_{\mathrm{T}}$ in the nonpsychotic group, we did not observe such a correlation among psychosis patients. Cognitive deficits in the patient group may thus reflect morbid factors such as, disease progression, protective effects of supportive care, and the effects of medications, medical, and psychiatric comorbidity and substance use, that are not predicted by $\mathrm{PRS}_{\mathrm{SCZ}}$. However, recently, a similar result was reported in a study of Autism Spectrum Disorder (ASD) in which the polygenic risk of ASD did not predict IQ in the ASD probands (despite a strong positive correlation in the general population ${ }^{61}$ ), although the polygenic scores of educational attainment and schizophrenia $\operatorname{did}^{62}$. This too could be due to factors other than the genetic risk of developing the disease playing a significant role in determining the pathologic trajectory of cognitive function in the ASD patients.

Due to the relatively small sample size of the B-SNIP cohort, one might be concerned about statistical power of these analyses. In the B-SNIP cohort $80 \%$ statistical power corresponded to a correlation of magnitude 0.2 in the PSYCH group $(N=314)$ and of 0.17 in the NPSYCH group at $P_{\mathrm{FDR}}=0.0064$. The $\mathrm{PRS}_{\mathrm{SCZ}}$ used here now explains $\sim 20 \%$ or more of case-control variation in schizophrenia risk $^{19}$, but it is difficult to estimate the expected relationship between that PRS and the specific domains of cognition considered here. It is particularly difficult because domains of cognition likely differ in their relationships with schizophrenia ${ }^{25,39}$. The genetic associations to the BACS should be replicated in a larger sample.

The polygenic score for educational attainment

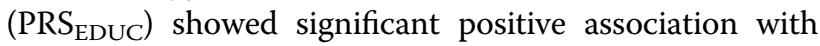
years of education in both the psychotic and the nonpsychotic groups in B-SNIP, and also showed significant association with WRAT in both B-SNIP and PNC. However, the association between $\mathrm{PRS}_{\mathrm{EDUC}}$ and BACS was not significant in the nonpsychotic group and was nominally significant $(p<0.05$, Table $\mathrm{S} 5)$ in the psychotic group in B-SNIP. While this lack of significant association with BACS in our work could be due to the relatively small B-SNIP sample size (BACS was not available in $\mathrm{PNC}$ ), it is also possible that different cognitive domains have varying degrees of genetic overlap with educational attainment, and that cognitive phenotypes that assess verbal abilities are more closely genetically linked to educational performance. For example, in the UK Biobank data Hagenaars et al. ${ }^{25}$ reported strong genetic correlation between educational attainment and verbal-numerical reasoning $\left(r_{\mathrm{g}} \sim 0.72\right)$, but the genetic correlation of educational attainment with memory and reaction time were not significant.

Our results indicate the need to further explore the relationship between cognitive performance and the genetic risk factors of psychiatric disorders in larger patient groups. These findings also suggest that specific domains of cognition may be more closely etiologically linked to schizophrenia than other domains are, creating an opportunity for longitudinal studies to identify the domains that best predict illness onset.

\section{Acknowledgements}

The B-SNIP study is in part supported by the National Institute of Mental Health (grants MH077852, MH077945, MH078113, MH077851, and MH077862). E.B.R. and D.J.W. were funded by National Institute of Mental Health grant $1 \mathrm{~K} 01 \mathrm{MH} 099286-01 \mathrm{~A} 1$ and Brain Behavior Research Foundation (NARSAD) Young Investigator grant 22379. Additional support was provided by the Stanley Center for Psychiatric Research at the Broad Institute of MIT and Harvard.

\section{Author details}

${ }^{1}$ Department of Genetics, Harvard Medical School, Boston, MA, USA. ${ }^{2}$ Stanley Center for Psychiatric Research, Broad Institute of MIT and Harvard, Cambridge, MA, USA. ${ }^{3}$ College of Physicians \& Surgeons, Columbia University Medical Center, New York, NY, USA. ${ }^{4}$ Department of Psychiatry and Cognitive Neurology, Beth Israel Deaconess Medical Center, Boston, MA, USA. ${ }^{5}$ Baylor College of Medicine, Texas Medical Center, Houston, TX, USA. ${ }^{6}$ Department of Psychiatry and Behavioral Neuroscience, University of Chicago, Chicago, IL, USA. ${ }^{7}$ Program in Medical and Population Genetics, Broad Institute of MIT and Harvard, Cambridge, MA, USA. ${ }^{8}$ Analytic and Translational Genetics Unit,

Department of Medicine, Massachusetts General Hospital and Harvard Medical School, Boston, MA, USA. ${ }^{9}$ Harvard Medical School, Boston, MA, USA.

${ }^{10}$ Department of Psychiatry, University of Pennsylvania, Philadelphia, PA, USA. ${ }^{11}$ Department of Psychiatry and Behavioral Sciences, Duke University Medical Center, Durham, NC, USA. ${ }^{12}$ Department of Psychology, Rosalind Franklin University of Medicine and Science, North Chicago, IL, USA. ${ }^{13}$ Department of Experimental and Clinical Pharmacology, University of Minnesota, Minneapolis, MN, USA. ${ }^{14}$ Department of Psychiatry, University of Minnesota, Minneapolis, MN, USA. ${ }^{15}$ Department of Psychology and Neuroscience, University of 
Georgia, Athens, GA, USA. ${ }^{16}$ Department of Psychiatry, University of Texas Southwestern Medical Center, Dallas, TX, USA. ${ }^{17}$ Department of Human Genetics, University of Chicago, Chicago, IL, USA. ${ }^{18}$ Departments of Psychiatry and Neurobiology, Yale University and Olin Neuropsychiatric Research Center, Hartford, CT, USA. ${ }^{19}$ Department of Psychiatry, Beth Israel Deaconess Medical Center and Massachusetts Mental Health Center, Harvard Medical School, Boson, MA, USA. ${ }^{20}$ Department of Psychiatry and Behavioral Neuroscience, University of Cincinnati, Cincinnati, OH, USA. ${ }^{21}$ Department of Epidemiology, Harvard T.H. Chan School of Public Health, Boston, MA, USA

\section{Conflict of interest}

R.S.E.K. receives royalties for the BACS. J.A.S. has been a consultant for Takeda Pharmaceuticals. C.A.T. is on the board of IntraCellular Technology and an ad hoc consultant for Takeda, Pierre Fabre, and Atiphony.

\section{Publisher's note}

Springer Nature remains neutral with regard to jurisdictional claims in published maps and institutional affiliations.

Supplementary Information accompanies this paper at (https://doi.org/ 10.1038/s41398-018-0124-8).

Received: 9 October 2017 Revised: 10 November 2017 Accepted: 15 January 2018

Published online: 12 April 2018

\section{References}

1. Kahn, R. S. \& Keefe, R. S. Schizophrenia is a cognitive illness: time for a change in focus. JAMA Psychiatry 70, 1107-1112 (2013).

2. Kraepelin, E. Psychiatrie: Ein Lehrbuch für Studierende und Ärtze. Verlag von Johann Ambrosius Barth, Leipzig, Germany, 1893).

3. Bilder, R. M. et al. Neuropsychology of first-episode schizophrenia: initial characterization and clinical correlates. Am. J. Psychiatny 157, 549-559 (2000).

4. Reichenberg, A. et al. Static and dynamic cognitive deficits in childhood preceding adult schizophrenia: a 30-year study. Am. J. Psychiatry 167, 160-169 (2010).

5. Liu, C. H., Keshavan, M. S., Tronick, E. \& Seidman, L. J. Perinatal risks and childhood premorbid indicators of later psychosis: next steps for early psychosocial interventions. Schizophr. Bull. 41, 801-816 (2015).

6. Khandaker, G. M., Barnett, J. H., White, I. R. \& Jones, P. B. A quantitative metaanalysis of population-based studies of premorbid intelligence and schizophrenia. Schizophr. Res. 132, 220-227 (2011).

7. McCabe, J. H. et al. Scholastic achievement at age 16 and risk of schizophrenia and other psychoses: a national cohort study. Psychol. Med. 38, 1133-1140 (2008).

8. Seidman, L. J. et al. Association of neurocognition with transition to psychosis: baseline functioning in second phase of the North American Prodome Longitudinal Study. JAMA Psychiatry 73, 1239-1248 (2016).

9. Seidman, L. J. et al. Neuropsychology of the prodrome to psychosis in the NAPLS consortium: relationship to family history and conversion to psychosis. Arch. Gen. Psychiatry 67, 578-588 (2010).

10. Fusar-Poli, P. et al. Cognitive functioning in prodromal psychosis: a metaanalysis. Arch. Gen. Psychiatry 69, 562-571 (2012).

11. Simon, A. E. et al. Cognitive functioning in the schizophrenia prodrome. Schizophr. Bull. 33, 761-777 (2007).

12. Sullivan, P. F., Kendler, K. S. \& Neale, M. C. Schizophrenia as a complex trait: evidence from a meta-analysis of twin studies. Arch. Gen. Psychiatry 60 , 1187-1192 (2003).

13. Bouchard, T. J. Jr. Genes, evolution and intelligence. Behav. Genet. 44, 549-577 (2014).

14. Haworth, C. M. et al. The heritability of general cognitive ability increases linearly from childhood to young adulthood. Mol. Psychiatry 15, 1112-1120 (2015).

15. Deary, I. J., Penke, L. \& Johnson, W. The neuroscience of human intelligence differences. Nat. Rev. Neurosci. 11, 201-211 (2010).

16. Plomin, R. \& Deary, I. J. Genetics and intelligence differences: five special findings. Mol. Psychiatry 20, 98-108 (2010).
17. Davies, G. et al. Genetic contributions to variation in general cognitive function: a meta-analysis of genome-wide association studies in the CHARGE consortium ( $\mathrm{N}=$ 53949). Mol. Psychiatry 20, 183-192 (2015).

18. Davies, G. et al. Genome-wide association studies establish that human intelligence is highly heritable and polygenic. Mol. Psychiatry 16, 996-1005 (2011).

19. Schizophrenia working group of the Psychiatric Genomics Consortium. Biological insights from 108 schizophrenia-associated genetic loci. Nature 511, 421-427 (2014).

20. Toulopoulou, T. et al. Substantial genetic overlap between neurocognition and schizophrenia: genetic modeling in twin samples. Arch. Gen. Psychiatry 64, 1348-1355 (2007).

21. Toulopoulou, T. et al. Impaired intellect and memory: a missing link between genetic risk and schizophrenia? Arch. Gen. Psychiatry 67, 905-913 (2010).

22. Fowler, T., Zammit, S., Owen, M. J. \& Rasmussen, F. A population-based study of shared genetic variation between premorbid IQ and psychosis among male twin pairs and sibling pairs from Sweden. Arch. Gen. Psychiatry 69, 460-466 (2012)

23. Bulik-Sullivan, B. et al. An atlas of genetic correlations across human diseases and traits. Nat. Genet. 47, 1236-1241 (2015)

24. Hubbard, L. et al. Evidence of common genetic overlap between schizophrenia and cognition. Schizophr. Bull. 42, 832-842 (2016).

25. Hagenaars, S. P. et al. Shared genetic aetiology between cognitive functions and physical and mental health in UK Biobank $(N=112151)$ and 24 GWAS consortia. Mol. Psychiatry 21, 1624-1632 (2016).

26. Lencz, T. et al. Molecular genetic evidence for overlap between general cognitive ability and risk for schizophrenia: a report from the Cognitive Genomics consortium (COGENT). Mol. Psychiatry 19, 168-174 (2014).

27. Hill, S. K. et al. Neuropsychological impairments in schizophrenia and psychotic bipolar disorder: findings from the Bipolar-Schizophrenia Network on Intermediate Phenotypes (B-SNIP). Am. J. Psychiatry 170, 1275-1284 (2013).

28. Tamminga, C. A. et al. Bipolar and schizophrenia network for intermediate phenotypes: outcomes across the psychosis continuum. Schizophr. Bull. $\mathbf{4 0}$ S131-S137 (2014)

29. Keefe, R. S. et al. The brief assessment of cognition in schizophrenia: reliability, sensitivity and comparison with a standard neurocognitive battery. Schizophr. Res. 68, 283-297 (2014).

30. Kareken, D. A., Gur, R. C. \& Saykin, A. J. Reading on the wide range achievement test-revised and parental education as predictors of iq: comparison with the Barona formula. Arch. Clin. Neuropsychol. 10, 147-157 (1995).

31. Bright, P., Jaldow, E. \& Kopelman, M. D. The national adult reading test as a measure of premorbid intelligence: a comparison with estimates derived from demographic variables. J. Int. Neuropsychol. Soc. 8, 847-854 (2002).

32. Gladsjo, J. A., Heaton, R. K, Palmer, B. W., Taylor, M. J. \& Jeste, D. V. Use of oral reading to estimate premorbid intellectual and neuropsychological functioning. J. Int. Neuropsychol. Soc. 5, 247-254 (1999).

33. Davies, G. et al. Genome-wide association study of cognitive functions and educational attainment in UK Biobank $(N=112151)$. Mol. Psychiatry 21, 758-767 (2016).

34. Okbay, A. et al. Genome-wide association study identifies 74 loci associated with educational attainment. Nature 533, 539-542 (2016)

35. Gur, R. C. et al. Age group and sex differences in performance on a computerized neurocognitive battery in children age 8-21. Neuropsychology $\mathbf{2 6}$ 251 (2012).

36. Robinson, E. B. et al. The genetic architecture of pediatric cognitive abilities in the Philadelphia Neurodevelopmental Cohort. Mol. Psychiatry 20, 454-458 (2015).

37. Calkins, M. E. et al. The Philadelphia neurodevelopmental cohort: constructing a deep phenotyping collaborative. J. Child. Psychol. Psychiatry 56, 1356-1369 (2015).

38. Clementz, B. A. et al. Identification of distinct psychosis biotypes using brainbased biomarkers. Am. J. Psychiatry 173, 373-384 (2016).

39. Germine, L. et al. Association between polygenic risk for schizophrenia, neurocognition, and social cognition across development. Transl. Psychiatry $\mathbf{6}$ e924 (2016).

40. Purcell, S. et al. PLINK: a tool set for whole-genome association and population-based linage analyses. Am. J. Hum. Genet. 81, 559-575 (2007).

41. Chang, C. C. et al. Second-generation PLINK: rising to the challenge of larger and richer datasets. Gigascience 4, 7 (2015).

42. Anderson, C. A. et al. Data quality control in genetic case-control association studies. Nat. Protoc. 5, 1564-1573 (2010). 
43. 1000 Genomes Project Consortium. et al. An Integrated map of genetic variation from 1,092 human genomes. Nature 491, 56-65 (2012).

44. Williams, A. L., Patterson, N., Glessner, J., Hakonarson, H. \& Reich, D. Phasing of many thousands of genotyped samples. Am. J. Hum. Genet. 91, 238-251 (2012).

45. Howie, B., Fuchsberger, C., Stephens, M., Marchini, J. \& Abecasis, G. R. Fast and accurate genotype imputation in genome-wide association studies through pre-phasing. Nat. Genet. 44, 955-959 (2012).

46. Howie, B. N., Donnelly, P. \& Marchini, J. A flexible and accurate genotype imputation method for the next generation of genome-wide association studies. PLoS Genet. 5, e1000529 (2009).

47. Howie, B., Marchini, J. \& Stephens, M. Genotype imputation with thousands of genomes. G3 (Bethesda) 1, 457-470 (2011).

48. Marchini, J. \& Howie, B. Genotype imputation for genome-wide association studies. Nat. Rev. Genet. 11, 499-511 (2011).

49. Yang, J., Lee, S. H., Goddard, M. E. \& Visscher, P. M. GCTA: a tool for genomewide complex trait analysis. Am. J. Hum. Genet. 88, 76-82 (2011).

50. The International HapMap 3 Consortium. Integrating common and rare genetic variation in diverse human populations. Nature 467, 52-58 (2010).

51. Das, S. et al. Next-generation genotype imputation service and methods. Nat. Gen. 48, 1284-1287 (2016).

52. The 1000 Genomes project consortium. A global reference for human genetic variation. Nature 526, 68-74 (2015).

53. Benjamini, Y. \& Hochberg, Y. Controlling the false discovery rate: a practical and powerful approach to multiple testing. J. R. Stat. Soc. Ser. B 57, 289-300 (1995).
54. Deary, V. et al. Genetic contributions to self-reported tiredness. Mol. Psychiatry 23, 609-620 (2017). e-pub ahead of print 21 March 2017.

55. RStudio Team. RStudio: Integrated Development for R. (RStudio, Inc. Boston, MA, 2015.).

56. Tomlinson-Keasey, C. \& Little, T. D. Predicting educational attainment, occupational achievement, intellectual skill, and personal adjustment among gifted men and women. J. Educ. Psychol. 82, 442-455 (1990).

57. Trampush, J. W. et al. GWAS meta-analysis reveals novel loci and genetic correlates for general cognitive function: a report from the COGENT consortium. Mol. Psychiatry 22, 336-345 (2017).

58. Bulik-Sullivan, B. K., Loh, P. R., Finucane, H. K., Ripke, S. \& Yang, J. Schizophrenia working group of the psychiatric genomics consortium et al. Nat. Genet. 47, 291-295 (2015).

59. Dudbridge, F. Power and predictive accuracy of polygenic risk scores. PLoS Genet. 9, e1003348 (2013).

60. Riglin, L. et al. Schizophrenia risk alleles and neurodevelopmental outcomes in childhood: a population-based cohort study. Lancet Psychiatry 4, 57-62 (2017).

61. Clarke, T. K. et al. Common polygenic risk for autism spectrum disorder (ASD) is associated with cognitive ability in the general population. Mol. Psychiatry 21, 419-425 (2016)

62. Weiner, D. J. et al. Polygenic transmission disequilibrium confirms that common and rare variation act additively to create risk for autism spectrum disorders. Nat. Genet. 49, 978-985 (2017). 